\title{
Resección endoscópica por tunelización submucosa de un leiomioma de la unión gastroesofágica: reporte de un caso
}

\author{
Ricardo Mejía ${ }^{1}$, Joaquín Sharp², Josefina Sáez ${ }^{3}$ y Allan Sharp ${ }^{1}$
}

\section{Submucosal tunneling endoscopic resection of a gastroesophageal junction leiomyoma: Report of a case}

Introduction: Upper gastrointestinal submucosal tumors are potentially malignant lesions; so safe and efficient treatments are needed. In recent years, submucosal tunneling endoscopic resection (STER) has emerged as a novel therapeutic technique, with promising results. Case report: 47-year-old male patient, previously asymptomatic, who was found to have a subepithelial lesion at the gastroesophageal junction. Upper endoscopic ultrasound was compatible with a leiomyoma. He underwent STER to remove the tumor and recovered with any complications. The biopsy confirmed an esophageal leiomyoma. Discussion: STER has become a highly feasible and safe therapeutic option for submucosal tumors of the gastroesophageal junction. The challenge is to spread knowledge about this technique, to maximize patient's benefit.

Key words: endoscopic resection; submucosal tumor; submucosal tunnelization.

\section{Resumen}

Introducción: Los tumores submucosos del tracto gastrointestinal alto tienen potencial de malignidad y también pueden ser una carga para el paciente, por lo que se requiere disponer de un tratamiento seguro y eficaz. El surgimiento de la técnica de resección endoscópica por tunelización submucosa (STER) en los últimos años ha mostrado resultados prometedores. Caso clínico: Se presenta el caso de un hombre de 47 años asintomático, en quien, en el contexto de un estudio preoperatorio de cirugía bariátrica, se pesquisa una lesión subepitelial en la unión gastroesofágica. La endosonografía alta es compatible con un leiomioma esofágico. Se realiza STER con éxito y sin complicaciones; a las $48 \mathrm{~h}$ es dado de alta. El estudio histopatológico confirmó un leiomioma esofágico. Discusión: La técnica STER ha demostrado consistentemente ser segura y eficaz en el tratamiento de los tumores submucosos de la unión gastroesofágica. El desafío es difundir la técnica por el resto del país, para mayor beneficio de los pacientes.

Palabras clave: resección endoscópica; tumor submucoso; tunelización submucosa.

\section{Introducción}

Los tumores submucosos (TSM) del tracto gastrointestinal alto menores de $30 \mathrm{~mm}$ son considerados en su mayoría benignos ${ }^{1}$. Sin embargo, algunos de estos tumores tienen potencial de malignidad ${ }^{2}$. Tradicionalmente, se ha propuesto la resolución quirúrgica en aquellos TSM mayores que $30 \mathrm{~mm}$ o que sean sintomáticos, considerándose, en caso contrario, el seguimiento endoscópico ${ }^{3,4}$.

En los casos que requiriesen resección, se recurría hasta poco antes de 2010 a la cirugía abierta (toracotomía o laparotomía) como primera opción, o bien, en centros especializados, al abordaje toracolaparoscópico ${ }^{5}$. Con el advenimiento de las técnicas endoscópicas se logró una aproximación menos invasiva y más segura, con buenos resultados a largo plazo, aunque no exenta de complicaciones ${ }^{6}$.

Aquellos pacientes que no cumplan con la indicación quirúrgica deben, entonces, someterse a seguimiento endoscópico periódico, lo cual a largo plazo puede resultar una importante carga económica y psicológica. Esto crea la necesidad de nuevos procedimientos más seguros y eficaces que los ya
Departamento de Cirugía Digestiva, División de Cirugía, Pontificia Universidad Católica de Chile, Santiago, Chile.

¿Escuela de Pregrado, Facultad de Medicina, Pontificia Universidad Católica de Chile, Santiago, Chile.

${ }^{3}$ Programa de Cirugía General, Facultad de Medicina, Pontificia Universidad Católica de Chile, Santiago, Chile.

Recibido el 3 de enero de 2017, aceptado para publicación el 16 de febrero de 2017.

Correspondencia a: Ricardo Mejía rjmejiam@gmail.com 
disponibles, para de tal modo poder ofrecer a los pacientes una resolución definitiva oportuna.

En este contexto, el grupo chino liderado por $\mathrm{Xu}$ crea en 2010 la técnica de resección endoscópica por tunelización submucosa (STER, por sus siglas en inglés), como una propuesta para el tratamiento de los TSM originados en la muscular propia ${ }^{7}$. La seguridad y eficacia de esta técnica para el tratamiento de este tipo de tumores ha sido ya documentada durante los últimos años ${ }^{8}$.

En este artículo se presenta el caso de la resección de un leiomioma de la unión gastroesofágica (UGE) mediante la técnica STER, lo que constituye el primer reporte de la implementación de esta técnica en Chile.

\section{Caso clínico}

Paciente de género masculino de 47 años de edad, con obesidad mórbida, hipertensión arterial, resistencia a la insulina y dislipidemia, en tratamiento farmacológico. En el contexto del estudio preoperatorio para una cirugía bariátrica se le realiza una endoscopia digestiva alta, en la cual se pesquisa una lesión subepitelial de aproximadamente $15 \mathrm{~mm}$ de diámetro, ubicada $20 \mathrm{~mm}$ proximal a la UGE. La endosonografía confirma una lesión de $18 \mathrm{~mm} \times 8 \mathrm{~mm}$ de diámetro originada en la muscular propia, sugerente de un leiomioma. Tras completar el estudio con una tomografía computarizada que no mostraba lesiones asociadas, se ofrece al paciente resolución quirúrgica con la técnica STER.

El tiempo total de la cirugía, llevada a cabo bajo anestesia general, fue de 115 min y contempló las siguientes etapas (Figura 1; vídeo en material suplementario):

- Elevación de la mucosa esofágica $5 \mathrm{~cm}$ proximal a UGE, con Voluven e índigo carmín.

- Mucotomía y disección submucosa, realizadas con cuchillo endoscópico Flush Knife BT de 2,5 mm.

- Creación de túnel submucoso hasta identificar la lesión.

- Aislamiento y resección de la lesión.

- Hemostasia, con pinza Coagrasper.

- Cierre de la mucotomía con clips metálicos.

Tras las 24 primeras horas de postoperatorio en régimen cero, se realizó un estudio contrastado esófago-estómago-duodeno, el cual descartó filtración. A las $48 \mathrm{~h}$ fue dado de alta sin complicaciones. El estudio histopatológico diferido confirmó el diagnóstico de leiomioma (Figura 1).

\section{Discusión}

El advenimiento de nuevas técnicas endoscópicas ha permitido el desarrollo de procedimientos quirúrgicos cada vez más seguros y eficaces. En este sentido, la técnica STER, al conservar la integridad de la pared esofágica, ha logrado reducir aún más la incidencia de complicaciones propias de este tipo de intervenciones. Es eficaz para el tratamiento de los TSM y permite en una sola intervención su diagnóstico y resolución definitiva. Así lo demostraron los creadores de la técnica en su primera publicación al respecto, en 2012: en una serie de 15 pacientes con TSM de la muscular propia informaron una tasa de resección en bloc de 100\%; las únicas complicaciones reportadas fueron un neumotórax con enfisema subcutáneo en un paciente y un neumoperitoneo en otro, manejados ambos de forma conservadora. Tras meses de seguimiento, la ausencia de recidiva fue absoluta ${ }^{7}$.

En 2015, Zhou et al. publicaron un estudio prospectivo que evaluó la seguridad y eficacia de la técnica para el tratamiento de TSM de la UGE ${ }^{8}$. En una cohorte de 21 pacientes, informaron una tasa de resección completa del $100 \%$ y de resección en bloc del $85,7 \%$. La única complicación fue el enfisema subcutáneo, que ocurrió en el $42,9 \%$ de los pacientes, requiriendo uno de ellos drenaje percutáneo para su resolución. Tras un promedio de 6 meses después de la intervención no había recidivas. En 2016, Li et al. publicaron un estudio prospectivo de una cohorte de 20 pacientes con TSM de la UGE, en el que una vez más se reportó una tasa de resección en bloc del $100 \%{ }^{9}$. En cuanto a complicaciones, un paciente desarrolló neumoperitoneo y 3 pacientes desarrollaron neumotórax, 2 de los cuales requirieron pleurostomía. Es interesante que, al comparar los pacientes con TSM esofagocardiales $v s$ los cardiales y los gastrocardiales, se observa que la tasa de complicaciones es menor en los esofagocardiales, a la vez que el tiempo operatorio es significativamente más prolongado en los gastrocardiales. Esto constituye uno de los primeros indicios de que la ubicación del tumor puede ser importante para el éxito del procedimiento.

La disección endoscópica submucosa (ESD) es, temporalmente, la predecesora de la STER, por lo que cabe preguntarse cuáles son las ventajas, si las hay, de esta última sobre la primera. Un estudio que aborda esta interrogante es el publicado en 2013 por Wang et al., con un diseño retrospectivo y basado en una cohorte de 39 pacientes, 18 operados con STER y 21 con ESD ${ }^{10}$. Los resultados indican que el tiempo operatorio es significativamente menor 

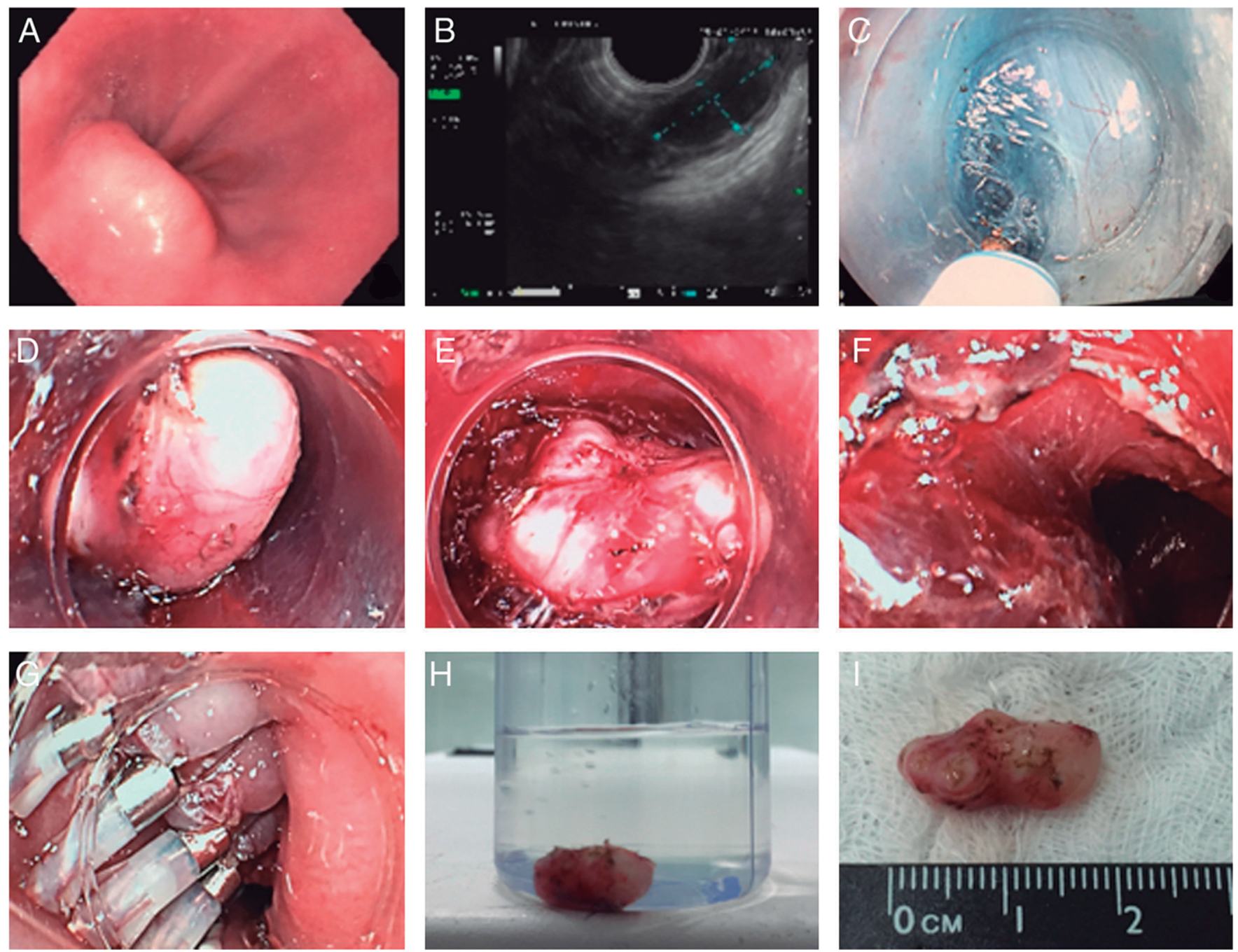

Figura 1. Técnica STER. A) Aspecto endoscópico de la lesión. B) Aspecto endosonográfico de la lesión, demostrando el probable origen en muscular propia. C) Creación del túnel submucoso. D) Tumor aislado en el túnel submucoso. E) Proceso de resección. F) Lecho de la lesión tras ser extraída. G) Mucotomía cerrada con clips. H) Aspecto de la lesión extraída. I) Medición del tumor, de $18 \mathrm{~mm}$ de diámetro mayor.

con STER que con ESD (67,5 min vs 87,2 min), al igual que la estadía hospitalaria (2,33 días vs 5,71 días), con igual eficacia y ambos sin recurrencias. La tasa de complicaciones fue mayor en el grupo ESD que en el STER ( $8 \%$ vs 3\%), pero sin diferencias significativas. Se concluye que la STER es una técnica segura y eficaz para el tratamiento de TSM de la UGE, con demostradas ventajas sobre la ESD.

En junio de 2016, Lv et al. publicaron la primera $\mathrm{y}$, hasta la fecha, única revisión sistemática sobre la STER, evaluando fundamentalmente su seguridad y eficacia ${ }^{11}$. El metaanálisis, que incluyó 28 estudios y 1.041 pacientes, informa los siguientes resulta- dos: tasa de resección completa de 97,5\%; tasa de resección en bloc de 94,6\%; incidencia de enfisema subcutáneo y/o neumomediastino de 14,8\%; incidencia de neumotórax de $6,1 \%$; incidencia de neumoperitoneo de $6,8 \%$; incidencia de perforación de $5,6 \%$. Se observa que la incidencia de enfisema subcutáneo y de neumomediastino es relativamente alta, más estas complicaciones son, por lo general, bastante inocuas. De este modo se concluye, con la evidencia de más alta calidad disponible hasta ahora, que la STER es una técnica altamente factible, eficaz y segura para el tratamiento de TSM del tracto gastrointestinal alto. Debe ser realizada por ciruja- 
nos o gastroenterólogos formados en endoscopia avanzada y con el equipo técnico apropiado.

Tras toda la evidencia discutida y con el reporte de este primer y exitoso caso, queda como desafío la difusión de la STER en el resto del país, de modo que los pacientes puedan beneficiarse y los profesionales capacitarse y/o conocer la disponibilidad de esta nueva técnica quirúrgica.

\section{Responsabilidades éticas}

Protección de personas y animales. Los autores declaran que para esta investigación no se han realizado experimentos en seres humanos ni en animales.

Confidencialidad de los datos. Los autores declaran que han seguido los protocolos de su centro de trabajo sobre la publicación de datos de pacientes.
Derecho a la privacidad y consentimiento informado. Los autores han obtenido el consentimiento informado de los pacientes y/o sujetos referidos en el artículo. Este documento obra en poder del autor de correspondencia.

\section{Conflicto de intereses}

Los autores declaran no tener ningún conflicto de intereses.

\section{Appendix A. Material adicional}

Se puede consultar material adicional a este artículo en su versión electrónica disponible en doi:10.1016/j.rchic.2017.02.006.

\section{Bibliografía}

1. Ponsaing LG, Kiss K, Hansen MB. Classification of submucosal tumors in the gastrointestinal tract. World $\mathbf{J}$ Gastroenterol. 2007;13:3311-5.

2. Lee IL, Lin PY, Tung SY, Shen CH, Wei $\mathrm{KL}, \mathrm{Wu} \mathrm{CS}$. Endoscopic submucosal dissection for the treatment of intraluminal gastric subepithelial tumors originating from the muscularis propria layer. Endoscopy. 2006;38:1024-8.

3. Hwang JH, Rulyak SD, Kimmey MB. American Gastroenterological Association Institute technical review on the management of gastric subepithelial masses. Gastroenterology. 2006;130:221728.

4. Demetri GD, von Mehren M, Antonescu CR, DeMatteo RP, GanjooKN, Maki RG, et al. NCCN Task Force Report: Update on themanagement of patients with gastrointestinal stromal tumors. J Natl Compr Canc Netw. 2010;8 Suppl. 2:S1-40.

5. Von Rahden BH, Stein HJ, Feussner H, Siewert JR. Enucleation of submucosal tumors of the esophagus: Minimally invasive versus open approach. Surg Endosc. 2004;18:924-30.

6. Shi Q, Zhong YS, Yao LQ, Zhou PH, Xu MD, Wang P. Endoscopic submucosal dissection for treatment of esophageal submucosal tumors originating from the muscularis propria layer. Gastrointest Endosc. 2011;74:1194-200.

7. Xu MD, Cai MY, Zhou PH, Qin XY, Zhong YS, Chen WF, et al. Submucosal tunneling endoscopic resection: A new techniquefor treating upper GI submucosal tumors originating from the muscularis propria layer (with videos). Gastrointest Endosc. 2012;75:195-9.

8. Zhou DJ, Dai ZB, Wells MM, Yu DL,
Zhang J, Zhang L. Submucosal tunneling and endoscopic resection of submucosal tumors at the esophagogastric junction. World J Gastroenterol.2015;21:578-83.

9. Li B, Liu J, Lu Y, Hao J, Liu H, Jiang J, et al. Submucosal tunneling endoscopic resection for tumors of the esophagogastric junction. Minim Invasive Ther Allied Technol. 2016;25:141-7.

10. Wang L, Ren W, Zhang Z, Yu J, Li Y, Song Y. Retrospective study of endoscopic submucosal tunnel dissection (ESTD) for surgical resection of esophageal leiomyoma. Surg Endosc. 2013;27:425966.

11. Lv XH, Wang CH, Xie Y. Efficacy and safety of submucosal tunneling endoscopic resection for upper gastrointestinal submucosal tumors: A systematic review and meta-analysis. Surg Endosc. 2017;31:4963, http://dx.doi. org/10.1007/s00464-016-4978-7. 